\title{
Substanzkonsum und Sterblichkeit: Das Dilemma der Kausalität
}

\author{
A. Uhl ${ }^{1}$ \\ L. Kraus ${ }^{2}$
}

\author{
Substance Use and Mortality: The Dilemma of Causality
}

\section{Zusammenfassung}

Anliegen: Durch Substanzkonsum verursachte vorzeitige Todesfälle und verlorene Lebensjahre spielen in der drogen- und suchtspezifischen Diskussion eine große Rolle. Der vorliegende Artikel setzt sich kritisch mit den empirischen und theoretischen Grundlagen der Berechnung dieser Maßzahlen auseinander, diskutiert, unter welchen Rahmenbedingungen diese praktisch relevant sowie sinnvoll interpretierbar sind, und macht auf einige grundlegende Probleme in Zusammenhang mit der Interpretation dieser Konzepte aufmerksam. Ergebnisse: Nur akute Todesfälle, die als Folge von Substanzkonsum unmittelbar auftreten, sind sinnvoll als substanzverursachte Todesfälle interpretierbar. Zur Quantifizierung langfristigerer Folgen des Substanzkonsums ist es zweckmäßiger, die Anzahl der verlorenen Lebensjahre zu erfassen. Infolge von Multikausalität sind in der Regel weder Todesfälle noch verlorene Lebensjahre über unterschiedliche Ursachen hinweg sinnvoll zu einem Gesamtwert addierbar. Schlussfolgerungen: Angesichts großer Unsicherheiten in Bezug auf Validität und Reliabilität der empirischen Grundlagen der Berechnung substanzbezogener Mortalitätsindikatoren ist bei der Interpretation von Mortalitätsmaßen große Vorsicht anzuraten. Außerdem sollte man sich mit den methodologischen Grundlagen und den logischen Problemen der Konzepte ausreichend vertraut machen, um gegen plausibel erscheinende, aber falsche Ad-hoc-Interpretationen gewappnet zu sein.

\section{Schliuisselwörter}

Substanzkonsum · Sterblichkeit · Todesursachen · Kausalität

\section{Abstract}

Concern: Cases of premature death and years of life lost due to substance use play a major role in discussions concerning substance use and addiction. This article looks critically into the empirical and theoretical foundation of the measures, discusses under what conditions they can be sensibly interpreted and are of practical relevance and puts the finger on basic problems related to the interpreting these concepts. Results: Only acute cases of death occurring causally related to substance use shortly after consumption may sensibly be interpreted as death caused by substance use. To assess longer-term causal effects of substance use on mortality it is more adequate to consider years of life lost. Due to multi-causality it makes little sense to add cases of premature death or years of life lost across different causes. Conclusions: Given enormous uncertainties in relation to validity and reliability of empirical data the calculations of substance related mortality indicators are based on, it is advisable to interpret those figures with great caution. Most important, persons interpreting the figures should be sufficiently familiar with the methodological foundation and with logical problems connected with the approach to prevent plausible but wrong ad hoc conclusions.

\section{Key words}

Substance use $\cdot$ mortality $\cdot$ causes of deaths $\cdot$ causality

Institutsangaben

${ }^{1}$ Ludwig-Boltzmann-Institut für Suchtforschung (LBISucht) und Anton-Proksch-Institut (API),

Wien, Österreich

2 IFT Institut für Therapieforschung, München

Korrespondenzadiresse

Dr. Alfred Uhl · Ludwig-Boltzmann-Institut für Suchtforschung (LBISucht) · Mackgasse 7 - 11 .

1230 Wien, Österreich · E-mail: Alfred.Uhl@api.or.at

Bibliografie

Suchttherapie 2006; 7: 162 - 172 @ Georg Thieme Verlag KG Stuttgart · New York

DOI 10.1055/s-2006-927287

ISSN 1439-9903 
Einleitung

Der Konsum von Alkohol, Tabak und illegalen Drogen steht ohne Zweifel in Zusammenhang mit einem erhöhten Risiko, vorzeitig zu sterben, an bestimmten Krankheiten zu erkranken, Verletzungen zu erleiden sowie Unfälle zu verursachen [1 -6]. In einer der meist zitierten Publikationen zu substanzbezogener Morbidität und Mortalität benennen English et al. [7] mehr als 60 Krankheiten, die nachweislich einen Bezug zu Substanzenkonsum aufweisen. Da man den langfristigen Einfluss des Substanzkonsums auf die Lebenserwartung von Menschen nicht in einem realen Experiment messen kann, ist es üblich, diesen Zusammenhang über Gedankenexperimente zu erfassen, welche die Realität einem kontrafaktischen Alternativszenario gegenüberstellen.

Bei gut geplanten randomisierten, kontrollierten Studien (randomised controlled trials, RCT) kann man über die Zufallsaufteilung der Probanden in eine Versuchs- und eine Kontrollgruppe (Randomisierung) Selbstselektionsmechanismen vor Beginn des Experiments ausschalten und im Sinne des Doppelblindansatzes systematische Veränderungen der Rahmenbedingungen während des Experiments (Durchführungsbias) weitgehend ausschalten. In nichtexperimentellen Settings kann man hingegen Verzerrungen durch unkontrollierte Drittvariablen (Confounder) nicht systematisch verhindern und muss versuchen, deren Einfluss durch Einbeziehung weiterer empirischer Befunde, durch mehr oder weniger gut fundierte bzw. mehr oder weniger plausible Annahmen sowie durch logisches Denken soweit wie möglich zu verringern. Forschung ähnelt hier dem Lösen eines unvollständiges Puzzles, bei dem fehlende Teile zunächst durch common sense und Logik ersetzt werden und längerfristig versucht wird, durch gezielte Forschungsschritte mehr Klarheit in die Sache zu bringen, bis sich letztlich ein einigermaßen gesichertes Bild der Realität abzeichnet [8].

Die Fülle der relevanten, aber nicht erfassten Drittvariablen sowie die Notwendigkeit, Zusatzannahmen einzuführen, macht die Resultate grundsätzlich vorläufig und unsicher und lässt viel Raum für Spekulation. Je komplexer die Konzepte zur Formulierung jener kontrafaktischer Szenarien sind, die als Grundlage für Gedankenexperimente dienen, und je höher der Aggregationsgrad der zugrunde liegenden empirischen Daten ist, desto schwerer wird es, logische Fehler zu identifizieren, zu belegen und zu kompensieren. Eine bewährte Möglichkeit, Fehler in derartigen Modellen zu entdecken, besteht darin, einfache oder extreme Situationen zu formulieren, an denen sich Widersprüche oder Unzulänglichkeiten anschaulich darstellen lassen. Um die Probleme hinter hoch aggregierten Daten identifizieren zu können, ist es ferner nötig, die Daten zumindest stichprobenartig bis zum Ursprung zurückzuverfolgen. Der vorliegende Beitrag greift beide Ansätze mit dem Ziel auf, den logischen Stellenwert und die empirische Fundierung unterschiedlicher Zugänge kritisch zu beleuchten.

Häufig sind Auftraggeber und Rezipienten wissenschaftlicher Studien nur wenig an der logischen und empirischen Fundierung von Koeffizienten und Maßzahlen interessiert, sondern primär an deren Verwertbarkeit. Die Medien lieben Zahlen, die bei Lesern, Hörern und Sehern Betroffenheit erzeugen. Sowohl Personengruppen, die von bestimmten Maßnahmen profitieren, als auch solche, die aus innerer Überzeugung für bestimmte Maß- nahmen eintreten, benötigen Zahlen, die die Notwenigkeit dieser Maßnahmen unterstreichen. Hier zählt primär die Plausibilität und Wirkung der Ergebnisse für Öffentlichkeit und Politik und weniger die wissenschaftliche Fundierung. Der vorliegende Artikel formuliert die Kritik daher massiver als üblich, in der Hoffnung, damit einen Anstoß zur kritischen Diskussion der Grundlagen und in der Folge zur Optimierung der wissenschaftlichen Zugänge zu bieten.

\section{Mortalitätskoeffizienten}

In der öffentlichen Diskussion über die Schädlichkeit und Gefährlichkeit bestimmter Substanzen bzw. Verhaltensweisen spielen Gesamtmaße zur Beschreibung des Gesundheitszustandes der Bevölkerung („summary measures of public health“ [9]) eine große Rolle. In diesem Zusammenhang geht es um eine Fülle ursachenbezogener Morbiditäts- bzw. Mortalitätskoeffizienten (vgl. dazu den Beitrag von Gmel und Rehm in diesem Heft). Der vorliegende Artikel beschäftigt sich ausschließlich mit ursachenbezogenen Mortalitätskoeffizienten (UMK); also mit der Frage, wie viele Todesfälle jeweils in einem ursächlichen Zusammenhang mit einem bestimmten Risikofaktor stehen (ursachenbezogene vorzeitige Todesfälle, UVT) oder wie viele Lebensjahre in Zusammenhang mit einem bestimmten Risikofaktor verloren gehen (ursachenbezogene verlorene Lebensjahre, UVL). Die primär interessierenden Risikofaktoren sind unterschiedliche Substanzkonsumgewohnheiten, die im Folgenden als „Exposition zu psychoaktiven Substanzen“ bezeichnet werden.

Die durch einen bestimmten Risikofaktor verursachten Todesfälle werden in der Literatur oft als „kausal attribuierbare Todesfälle“ (z.B. alcohol attributable deaths [10] oder nicotine attributable mortality [11]), „vorzeitige Todesfälle“ (cases of premature death [12]) oder „vermeidbare Todesfälle“ (avoidable mortality [7]) bezeichnet. Der Ausdruck ,vermeidbare Todesfälle“ ist dabei irreführend, weil der Tod ja grundsätzlich nicht vermieden, sondern bestenfalls aufgeschoben werden kann.

Der Anteil der durch einen Risikofaktor (z.B. Alkoholkonsum) zusätzlich verursachten Todesfälle an allen Verstorbenen bzw. an jenen Fällen, die an einer bestimmten Krankheit (z.B. Leberzirrhose) gestorben sind, wird als „kausal attribuierbarer Anteil“ (alcohol attributable fraction [13]) oder als „ursächlicher Anteil“ (etiologic fraction [7]) bezeichnet. Die Differenz zwischen dem Todeseintritt im Falle der Exposition zu einem Risikofaktor und der Lebenserwartung wird als auf diesen Risikofaktor „attribuierbare verlorene Lebensjahre“ (years of life lost to premature mortality [14]) bezeichnet. In der Folge verwenden wir die Ausdrücke KAA für den „kausal attribuierbaren Anteil“, UVT für „ursachenbezogene vorzeitige Todesfälle“ und UVL für „ursachenbezogene verlorene Lebensjahre“.

\section{Mortalitätskoeffizienten und Kausalität}

Die Maße UVT und UVL stellen einen Ursache-Wirkungs-Zusammenhang zwischen einem Risikofaktor und der Sterblichkeit her. Aus theoretischer Sicht wird eine Kausalbeziehung zwischen Sub- 
stanzkonsum und Tod vorausgesetzt und natürlich auch, dass der Ursache-Wirkungs-Zusammenhang messbar ist.

\section{Experimente oder Gedankenexperimente}

Der Königsweg, um Kausalität zu identifizieren und zu prüfen, sind randomisierte kontrollierte Studien (randomised controlled trials, RCT). In diesen Experimenten werden die Probanden zufällig einer Versuchs- und einer Kontrollbedingung zugeteilt, die relevanten Bedingungen werden über geeignete Maßnahmen manipuliert und potenziell störende Einflüsse während des Beobachtungszeitraums werden weitestgehend kontrolliert. Die Randomisierung der Probanden zu Beginn des Experiments soll einen Bias durch Selbstselektion und die Kontrolle der Rahmenbedingungen während des Experiments einen Durchführungsbias verhindern. Der kausal auf die Experimentalbedingung zurückführbare Effekt (KAA) wird dann beispielsweise als Differenz der Ergebnisvariablen in Versuchs- und Kontrollbedingung errechnet.

Im Rahmen epidemiologischer Fragestellungen gibt es aus ethischen, ökonomischen, technischen und ontologischen Gründen nur selten die Möglichkeit, reale Experimente durchzuführen. So kann man z. B. eine große Gruppe von Jugendlichen nicht zufällig in zwei Gruppen aufteilen, einer Gruppe über Jahrzehnte hinweg den Konsum einer bestimmten Menge Alkohol pro Tag sowie der anderen Gruppe völlige Alkoholabstinenz vorschreiben, dann noch nahtlos die Einhaltung der Instruktionen kontrollieren und auch garantieren, dass alle Rahmenbedingungen konstant bleiben.

Wo reale Experimente nicht möglich sind, lassen sich Gedankenexperimente durchführen, in denen anstelle der experimentellen Versuchssituation aus Daten und Annahmen eine kontrafaktische Referenzsituation konstruiert wird, die dann der realen Situation gegenübergestellt wird [15]. Je näher das kontrafaktische Szenario einem randomisierten kontrollieren Experiment kommt, desto verlässlicher und relevanter sind die Ergebnisse. Es ist daher notwendig, die Analogie zum realen Experiment immer mitzudenken.

\section{Berechnung von UVT und UVL}

Im Experiment kann man nur solche Bedingungen ändern, die durch konkrete Maßnahmen tatsächlich herstellbar sind. Daraus folgt, dass man im Experiment nicht einfach Bedingungen ändern kann, die unveränderbar sind, und dass man, abgesehen von den erwarteten Auswirkungen, in der Regel immer auch mit unerwarteten und unerwünschten Effekten (Nebenwirkungen) rechnen muss. Reale Experimente sind daher Interventionsanalysen und können sich nicht beliebig von der Realität lösen. Im Gedankenexperiment sind der Fantasie dagegen keine Grenzen gesetzt. Man kann über Interventionsanalysen hinaus Bedingungen, unabhängig von der Frage, mit welchen Maßnahmen man diese verändern könnte, einfach umdenken und man kann Nebenwirkungen im Gedankenexperiment einfach ausblenden. Bei der Berechnung von UVT und UVL wird, wie z.B. Murray et al. $[16,17]$ betonen, nicht nur auf potenziell vermeidbare Folgen, sondern durchaus auch auf potenziell unvermeidbare kausal attribuierbare Folgen abgezielt.
Grundsätzlich ist nichts dagegen einzuwenden, rein fiktive Gedankenexperimente ohne praktische Relevanz anzustellen. Wenn das der Fall ist, sollte dies allerdings deutlich kenntlich gemacht werden. Man könnte beispielsweise abschätzen, welche positiven Effekte ein hochgradig lebertoxisches Medikament hätte, wenn die unerwünschten Nebenwirkungen nicht wären. Da unerwünschte Nebenwirkungen in den seltensten Fällen vollständig zu vermeiden sind, ist dieser Ansatz nur dann praktisch relevant, wenn man die positiven und die negativen Effekte gegeneinander aufrechnet. Beispielsweise sind „Krankheitskostenanalysen“, die Krankheiten einfach wegdenken, ohne konkrete Maßnahmen zur Erzielung dieser Bedingungen zu nennen und ohne zu bedenken, mit welchen indirekten Auswirkungen man rechnen müsste, nicht wirklich zweckmäßig [18]. Es ist nur wenig hilfreich, festzustellen, dass durch die Summe aller Erkrankungen und Unfallfolgen unendlich viele potenzielle Lebensjahre verloren gehen. Praktisch relevant ist dagegen die Feststellung, wie viele potenzielle Lebensjahre durch geeignete Maßnahmen gewonnen werden könnten und welche, unter Umständen negative, Konsequenzen im Gegenzug akzeptiert werden müssten. Mooney et al. [19] sprechen sich für Interventionsanalysen und gegen Krankheitskostenanalysen mit dem Argument aus, dass sich Forschungsanstrengungen angesichts begrenzter Ressourcen auf praktisch relevante Fragen konzentrieren sollten.

\section{Die Verwendung von Begriffen wie „assoziiert“ oder „bezogen“}

Kausalität manifestiert sich zwar oft in Assoziationen bzw. Korrelationen zwischen Ursache und Wirkung, das muss aber nicht so sein. Eine Kausalbeziehung kann einerseits durch Drittvariablen (confounder) völlig kompensiert oder sogar ins Gegenteil gekehrt werden, und wo kein Kausalzusammenhang vorliegt, kann anderseits über Drittvariablen ein Scheinzusammenhang entstehen. Es steht außer Frage, dass starke Luftverschmutzung bestimmte Atemwegserkrankungen begünstigt und so die Lebenserwartung verringert. Dennoch ist in vielen Regionen trotz kontinuierlich steigender Luftverschmutzung die Lebenserwartung über Jahrzehnte erheblich gestiegen. Das ist bei Berücksichtigung relevanter Drittvariablen leicht erklärbar. In jenen Regionen, in denen Wohlstand, Sicherheit und medizinische Versorgung enorm gestiegen sind, hat auch das Ausmaß der Luftverschmutzung kontinuierlich zugenommen. Die positiven Wohlstandseffekte haben die negativen Effekte der Luftverschmutzung auf die Gesundheit aber mehr als ausgeglichen. Aus diesem Grund ist es unzulässig, Assoziationen als Beweis für einen Kausalzusammenhang zu werten, auch wenn ein Zusammenhang - im Gegensatz zu obigem Beispiel - sehr plausibel erscheint. Anschaulich zeigt sich dieser logische Fehler, wenn man aus dem Zusammenhang zwischen Luftverschmutzung und der gleichzeitig steigenden Lebenserwartung schließt, dass durch die Förderung von Luftverschmutzung die Lebenserwartung erhöht werden könnte.

Dass Kausalität nicht aus Korrelationen abgeleitet werden kann, steht zwar in jedem einführenden Statistikbuch und ist auch allen empirisch arbeitenden Forschern ein zentrales Dogma, wird aber immer wieder vergessen, wenn Daten, die man für Kausalaussagen benötigen würde, nicht vorliegen, und wenn die Öffentlichkeit von Experten aber genau solche Kausalaussagen erwartet. Hier 
gibt es eine einfache, wenngleich nicht wirklich seriöse Lösung, nämlich Assoziationen mit Ausdrücken wie „substanzassoziiert“ oder „substanzbezogen“ in einer Art und Weise zu beschreiben, die einen Kausalzusammenhang suggeriert, ohne explizit den Begriff „substanzbedingt“ zu verwenden. In diesem Sinne wird das Kausalitätsproblem in Zusammenhang mit nichtexperimentellen Daten verschleiert, anstatt es zum zentralen Gegenstand methodologischer Reflexion zu machen [20].

\section{Die Verwendung von UVT und UVL}

\section{Kurzfristige Effekte}

Manche Risikobedingungen wirken sich auf die Mortalität akut und manche langfristig aus. Langfristige Effekte erfordern einen langen Beobachtungszeitraum. An einem fiktiven Experiment kann man demonstrieren, dass die Berechnung der UVT mit wachsendem Beobachtungszeitraum immer stärker von der Realität abweicht. Wenn man im Rattenexperiment mit 40 Kontrollratten und 40 Versuchsratten eine hochgiftige Substanz $\mathrm{X}$ in einer Dosierung testet, die bei $50 \%$ der Tiere rasch zum Tod führt, kann man den Beobachtungszeitraum so kurz ansetzen, dass mit großer Wahrscheinlichkeit keine der verbleibenden 20 Ratten in der Versuchsbedingung und keine der 40 Ratten in der Kontrollbedingung verstirbt. Dies ergäbe einen auf die Substanz X kausal attribuierbaren Anteil (KAA) von $100 \%$ und man würde alle 20 toten Ratten korrekterweise als Opfer der Substanz X klassifizieren (zur Berechnung attributabler Anteile vgl. den Beitrag von Gmel und Rehm in diesem Heft). Wäre nun durch einen unglücklichen Zufall in der Kontrollbedingung eine Ratte gestorben, so würde sich immerhin noch ein KAA von 90,5\% ergeben und man würde 19 der 21 verstorbenen Ratten kausal der Substanz X zuschreiben.

Würde man aber fälschlich erwarten, dass die Substanz X langfristig wirkt, und den Beobachtungszeitraum so lang wählen, dass z.B. in der Kontrollgruppe die Hälfte der Tiere (20 Ratten) eines natürlichen Todes gestorben wäre, so würde man dann in der Versuchsgruppe 30 tote Tiere zählen, nämlich 20 aufgrund der Giftwirkung verstorbene und 10 eines natürlichen Todes gestorbene ${ }^{1}$. Würde man nun - den wahren Sachverhalt nicht kennend - den kausal auf die Substanz attribuierbaren Anteil an den insgesamt verstorbenen 50 Tieren mittels Differenzbildung eruieren (in der Versuchsgruppe sind 10 Tiere mehr gestorben), käme man auf einen KAA von 20\%. Damit würde man den tatsächlichen Effekt der Substanz X um die Hälfte unterschätzen. Statt 20 würde man nur 10 verstorbene Tiere der Wirkung der Substanz X zuschreiben.

Würde man schließlich den Beobachtungszeitraum so weit verlängern, dass in beiden Gruppen alle Tiere an der Substanz oder natürlich gestorben wären, ergäbe sich ein KAA von $0 \%$, und man käme zur unzutreffenden Überzeugung, dass die Substanz X keinen Einfluss auf die Lebenserwartung der Ratten habe. Hätte man sich der Fragestellung jedoch nicht über die Berechnung der UVT, sondern der UVL angenähert, würde man in allen drei Fällen zu sinnvoll interpretierbaren Ergebnissen gelangen.

\section{Langfristige Effekte}

Die Zahl der UVT erfasst die Mortalität qualitativ und verzichtet auf jegliche quantitative Information. Bei dieser Maßzahl macht es z.B. keinen Unterschied, ob 30\% der Bevölkerung infolge geringfügig suboptimaler Ernährungsgewohnheiten jeweils einen Tag vorzeitig sterben oder ob infolge eines gravierenden Risikofaktors 30\% der Bevölkerung um durchschnittlich 20 Jahre früher sterben. Dazu kommt erschwerend, dass man auch bei einem gravierenden Risikofaktor nicht ausschließen kann, dass dieser für einen Teil der Fälle lebensverlängernd wirken könnte. In diesem Fall müsste man bei der Berechung der UVT eigentlich die Zahl jener, die vorzeitig sterben, mit der Zahl jener, die länger leben, gegenrechnen, was aber nicht passiert. Berechnet man aber stattdessen die UVL, wird dieses Problem implizit adäquat gelöst.

\section{Schätzung ursachenbezogener Todesfälle}

\section{Direkte und indirekte ursachenbezogene Todesfälle}

Generell wird zwischen „direkten UVT“ und „indirekt geschätzten UVT“ unterschieden [13]:

- Als direkte UVT gelten Tote in jenen Situationen, in denen von Beobachtern alle Fälle zu 100\% einem bestimmten Risikofaktor zugeschrieben werden. Ezzati et al. [14] nennen diesen $\mathrm{Zu}$ gang auch kategoriellen Ansatz. Der auf den Risikofaktor KAA wird dabei mit $100 \%$ angesetzt.

- Als indirekt geschätzte UVT gelten jene Fälle, bei denen der KAA über einen Vergleich zwischen einer Versuchsbedingung und einer Kontrollbedingung oder im Falle des Gedankenexperiments aus dem Vergleich zwischen einem kontrafaktischen Szenario und der Realität geschätzt wird. Ezzati et al. [14] sprechen in diesem Zusammenhang vom kontrafaktischen Ansatz.

Als direkte UVT in Zusammenhang mit Alkohol gelten z. B. Alkoholunfälle, alkoholbedingte Leberzirrhosen, Aggressionsdelikte unter Alkoholeinfluss oder Selbstmorde von Alkoholikern. Die mechanistische Klassifizierung bestimmter Todesursachen als $100 \%$ kausal auf eine bestimmte Substanz attribuierbar hält einer logischen Analyse allerdings keinesfalls stand. So wird das tatsächliche Ausmaß oft erheblich überschätzt. Anschaulich lässt sich das am Beispiel von Alkoholunfällen im Straßenverkehr darstellen.

\section{Alkoholunfälle im Straßenverkehr}

Als alkoholbedingte Verkehrsunfälle werden international jene Unfälle bezeichnet, bei denen ein beteiligter Lenker, Beifahrer oder Fußgänger, unabhängig von der Verschuldensfrage, alkoholisiert war. Das inkludiert nicht nur Unfälle, bei denen eine Mitschuld von alkoholisierten Beteiligten nicht eindeutig ausgeschlossen werden kann, sondern auch Fälle, wo eine Mitschuld gar nicht in Frage kommt. Das wäre z.B. ein Unfall, bei dem ein alkoholisierter Fahrzeuglenker, der vorschriftsmäßig vor einer roten Ampel stehen bleibt, von einem nüchternen Fahrzeuglenker gerammt wird, oder Unfälle mit ausschließlich nüchternen Lenkern, bei denen alkoholisierte Beifahrer verletzt wer-

\footnotetext{
1 Zehn ist die Hälfte der zunächst überlebenden 20 Tiere.
} 
den². Hinzuzählen zu den so erfassten Alkoholunfällen müsste man noch eine gewisse Dunkelziffer, weil bei schwer verletzten und/oder tödlich verunglückten Personen oft kein Alkoholtest durchgeführt wird und in solchen Fällen der Unfall nur dann als Alkoholunfall gewertet werden kann, wenn andere eindeutige Hinweise auf Alkoholisierung der Person vorliegen.

Es ist unmittelbar einzusehen, dass man nicht alle genannten Alkoholunfälle plus Dunkelziffer als durch Alkoholabstinenz vermeidbare Unfälle auffassen darf, weil logischerweise durch Alkoholabstinenz jene so genannten „Alkoholunfälle“ nicht vermieden werden können, bei denen der Verursacher ohnedies nüchtern war. Aber selbst wenn man nur jene Unfälle berücksichtigen würde, bei denen die Verursacher alkoholisiert waren, und diese Unfälle zu 100\% auf Alkohol attribuiert, überschätzt man den Einfluss des Alkohols systematisch, weil ja auch völlig nüchterne Personen Verkehrsunfälle verursachen. Der auf Alkohol KAA entspricht also der Zahl der erfassten Alkoholunfälle plus Dunkelziffer, vermindert um jene Alkoholunfälle, bei denen die alkoholisierten Beteiligten gar keinen Einfluss auf das Unfallgeschehen hatten, und vermindert um jenen Anteil, der den alkoholisierten Unfallverursachern auch im nüchternen Zustand passiert wäre. Auszuschließen ist übrigens auch nicht, dass jemand aufgrund seiner Alkoholisierung einen Unfall vermeidet, weil er z. B. - wissend um seinen Zustand und die damit verbundene Gefahr - besonders vorsichtig und langsam oder mit einem öffentlichen Verkehrsmittel fährt.

An einer korrekten Berechnung des KAA an den Alkoholunfällen besteht aus nachvollziehbaren Gründen nur wenig Interesse, da Alkohol am Steuer zweifelsohne ein ernsthaftes zusätzliches Unfallrisiko darstellt und die bloße Zahl von Alkoholunfällen als hinreichendes Präventionsargument gesehen wird. Aus wissenschaftlicher Perspektive wäre ein sachlich korrekter Zugang, auch wenn das zu erheblich niedrigeren Zahlen führen könnte, allerdings sehr zu begrüßen.

Mit einigem Aufwand ist es durchaus möglich, den ursächlichen Einfluss von Alkohol auf Straßenverkehrsunfälle und Verkehrstod zu schätzen, beispielsweise indem man, wie das Borkenstein et al. [22] taten, eine aufwändige Roadside-Case-Control-Studie durchführt. Bei diesem als "Grand-Rapids-Studie“ bekannten Forschungsprojekt wurden möglichst rasch nach jedem Verkehrsunfall einige weitere Fahrzeuge aufgehalten, sowohl bei den am Unfall beteiligten als auch bei den nachfolgenden, am Unfall unbeteiligten Verkehrsteilnehmern Alkoholtests durchführt und auch noch relevante Drittvariablen wie Geschlecht, Alter, Fahrpraxis etc. erhoben. Wenn man nämlich den Alkoholisierungsgrad der an Unfällen beteiligten Personen und jenen von - nach Fahrpraxis, Alter, Geschlecht etc. - vergleichbaren, nicht an Unfällen beteiligten Verkehrsteilnehmern unter identischen Bedingungen (Ort, Zeit, Witterungsverhältnisse etc.) kennt, so kann man das erhöhte

\footnotetext{
2 Es ist anzunehmen, dass es bei der Erfassung der Alkoholunfälle hinsichtlich Regeln und praktischer Handhabung relevante Unterschiede zwischen unterschiedlichen Staaten gibt. In Österreich gilt z. B. ein Unfall, bei dem ausschließlich ein Beifahrer alkoholisiert war, nur dann als Alkoholunfall, wenn der Beifahrer entweder verletzt wurde oder anzunehmen ist, dass er durch sein Verhalten am Zustandekommen des Unfalls beteiligt war [21].
}

Risiko unter Alkoholeinfluss errechnen und darauf aufbauend relativ verlässlich schätzen, wie viele der Alkoholunfälle selbst dann passiert wären, wenn die Beteiligten zum Unfallzeitpunkt völlig nüchtern gewesen wären.

\section{Aufsummieren der attribuierbaren Anteile von Todesursachen}

Beim indirekten Ansatz zur Berechnung der UMK gibt es grundsätzlich zwei Zugänge: Man kann die Zahl der UVT aus der Differenz zwischen der realen Situation und der kontrafaktischen Situation direkt schätzen und man kann die einzelnen Todesursachen aus der Sterbestatistik mit dem für diese Todesursache typischen KAA multiplizieren und dann die Einzelergebnisse aufaddieren (all cause mortality oder individual conditions). English et al. [7] fordern in diesem Zusammenhang die Berechnung über beide Ansätze, da, wie von MacMahon [23] gezeigt werden konnte, beim Aufsummieren der Einzelergebnisse das Gesamtausmaß überschätzt werden kann.

\section{Monokausalität vs. Multikausalität}

Alle Phänomene, mit denen wir zu tun haben, sind multikausal verursacht, in dem Sinn, dass sie nur entstehen können, wenn eine große Anzahl von notwendigen Bedingungen - man könnte hier von theoretischen Einzelursachen sprechen - erfüllt ist. So erfordert z.B. das Leuchten einer Stehlampe im Wohnzimmer unter anderem, dass eine Stehlampe und ein Wohnzimmer vorhanden sind, dass jede kleinste Komponente in der Stromversorgungskette vom Kraftwerk bis zur Glühbirne funktioniert und vieles mehr.

Es ist allerdings nicht immer nötig, sich dem Problem der Multikausalität im Einzelfall zu stellen. Wenn eine Stehlampe plötzlich nicht funktioniert, weil die Glühbirne durchgebrannt ist, so gibt es aus einer Erkenntnisperspektive zwar eine unbegrenzte Zahl von theoretischen Einzelursachen, die das Durchbrennen der Glühbirne zu diesem Zeitpunkt erklären - die Erkenntnis über diese Ursachen kann erheblich zur Optimierung zukünftiger Glühbirnen beitragen -, aber aus der praktischen Perspektive des Elektrikers, der den Defekt bloß beheben soll, reicht es in der Regel völlig aus, das Durchbrennen der Glühbirne als praktisch relevante konkrete Einzelursache zu interpretieren. Er kann die Frage, warum die Glühbirne letztlich durchgebrannt ist, ausblenden und durch Austauschen der Glühbirne die Funktionalität der Stehlampe wiederherstellen. Sollte allerdings, um beim Beispiel zu bleiben, die Glühbirne immer wieder nach unüblich kurzer Zeit durchbrennen, so ist es auch für den Elektriker zweckmäßig, sich aus der begrenzten praktischen Perspektive der konkreten Ursachen zu erheben und nach weiteren Erkenntnissen über Ursachen des Phänomens zu forschen.

Eine häufig angewandte Strategie, Multikausalität auf Monokausalität zu reduzieren, besteht darin, nach der „eigentlichen“ Ursache zu suchen; konkreter formuliert heißt das, das Problem aus einer ethischen Perspektive zu betrachten und nach einem Schuldigen zu suchen. Wenn ein Techniker eine Blitzschutzanlage nicht ordnungsgemäß regelmäßig wartet und irgendwann ein Blitz einschlägt, fünf Komponenten im elektrischen System zerstört werden und die Stehlampe daher nicht mehr funktioniert, würde kaum jemand zögern, die Unterlassung des Technikers als eigentliche Ursache für den Defekt zu bezeichnen. Kann man keine schuldige Person finden, weil z.B. die Blitzschutzanlage 
ordnungsgemäß gewartet wurde, wird einfach der Blitz zum Schuldigen erklärt.

Am Beispiel des Blitzschlages kann man die Implikationen der unterschiedlichen Perspektiven unmittelbar erkennen. Aus einer Erkenntnisperspektive gibt es unendlich viele theoretische Einzelursachen, die dazu geführt haben, dass der Defekt auftreten konnte. Aus der praktischen Perspektive des Elektrikers gibt es fünf konkrete Ursachen, die er beseitigen muss, um nach dem Blitzschlag die Funktionalität der Lampe wiederherzustellen. Aus der ethischen Perspektive der meisten Menschen gibt es dagegen meist nur eine einzige eigentliche Ursache: je nach Erkenntnisstand, die Unterlassung des Technikers oder den Blitzschlag.

Wenn, um zum eigentlichen Thema zurückzukehren, psychoaktive Substanzen als Schuldige für unterschiedlichste Probleme dargestellt werden, wenn diese im Rahmen einer ethischen Perspektive zu eigentlichen Ursachen personifiziert werden, tritt zwangsläufig ein Reduktionismus in Kraft, der multikausalen Komplexität hin zu einer monokausalen Betrachtung. Nur aus dieser Perspektive heraus ist es verständlich, wieso es in Öffentlichkeit und Wissenschaft so wenige Einwände dagegen gibt, Unfälle mit Alkoholbeteiligung, die Mehrzahl der Leberzirrhosen, Aggressionsdelikte unter Alkoholeinfluss, Selbstmorde von Süchtigen etc. ohne Zögern grundsätzlich und ausschließlich als „alkoholverursacht“ zu interpretieren.

\section{Genetik vs. Umwelt}

Aus der dargestellten Problematik von Monokausalität und Multikausalität folgt unmittelbar, dass man unterschiedliche Ursachen für ein Phänomen aus einer erkenntnisorientierten wissenschaftlichen Perspektive nie und aus einer praktisch lösungsorientierten Perspektive nur dann addieren kann, wenn tatsächlich Monokausalität gegeben ist. Wenn eine Addition nicht sinnvoll ist, ist es auch nicht sinnvoll, die Ursachen am Zustandekommen des Phänomens in Prozentanteile einzuteilen. Um es pointiert zu formulieren: Es ist sinnlos zu fragen, welchen Anteil am Gelingen eines Kuchens der Herd, welchen Anteil die Backform und welchen der Teig hat. Jede Komponente ist unbedingt nötig und somit zu 100\% unverzichtbar.

Genau aus diesem Grund ist es, wie Rothman und Greenland [15] belegen, auch nicht sinnvoll zu fragen, welchen Anteil am Zustandekommen bestimmter Krankheiten die Umwelt und welchen Anteil die Umgebung hat, auch wenn diese Vorgangsweise über eine inadäquate Analogie zu Tortenstücken oder zur Varianzzerlegung in der Statistik plausibel erscheint. Beispielsweise ist die Entstehung von AIDS zu 100\% genetisch bedingt, weil man ohne das entsprechende Erbgut die Krankheiten gar nicht entwickeln könnte, und gleichzeitig zu 100\% umweltbedingt, weil die Krankheit eine Exposition zum HI-Virus voraussetzt. Addiert man den Einfluss von Vererbung und Umwelt auf Krankheiten, so erklärt man einen Anteil von $200 \%$, was zu Recht seltsam anmutet. Das Resultat auf $100 \%$ zu beschränken, also der Umwelt und der Vererbung jeweils genau die Hälfte am Zustandekommen von Krankheiten zuzuweisen, würde die Groteske zwar besser verschleiern, aber nichts an der grundlegenden Problematik ändern.

Rothman und Greenland [15] kritisieren in diesem Zusammenhang Doll und Peto [24], die eine Liste von Krebsursachen mit
Prozentangaben anbieten, deren Summe sich annähernd auf $100 \%$ beläuft, was ungerechtfertigterweise Monokausalität und eine fast vollständige Aufklärung der Ursachen suggeriert. Pointiert formuliert: Wenn Monokausalität nicht gegeben ist und 50 Kausalfaktoren nachgewiesen werden, die jeweils $2 \%$ einer Erkrankung verursachen, so kann man zwar sagen, dass man durch Ausschaltung eines Faktors durchschnittlich $2 \%$ der Krebsfälle verhindern könnte, aber es könnte theoretisch auch sein, dass man durch Ausschaltung der weiteren 49 Faktoren keinen einzigen weiteren Krebsfall verhindern könnte, weil sich alle Ursachen auf die gleichen $2 \%$ der Krebsfälle beziehen.

\section{Additivität von UVT und UVL pro Ursache}

Die Frage, ob UVT und UVL pro Ursache additiv sind, lässt sich in einem Gedankenexperiment beantworten. Angenommen, wir beobachten eine homogene Teilpopulation Depressiver, die ihre Depressionen mit Substanzmissbrauch bekämpfen und irgendwann eine an sich nicht tödliche Dosis Heroin in Verbindung mit einer an sich nicht tödlichen Alkoholdosis konsumieren und das Bewusstsein verlieren. Nehmen wir ferner an, die Betreffenden hätten bei adäquater medizinischer Versorgung zwar grundsätzlich gerettet werden können, sterben aber, weil die Anwesenden Angst hatten, den Notarzt zu rufen. Nehmen wir darüber hinaus noch an, diese Personen hätten durchschnittlich um 10 Jahre länger gelebt, wenn sie nicht an der Überdosierung vorzeitig gestorben wären. Das ergibt jeweils vier Kausalursachen für jeden Todesfall. Wäre die Depression rechtzeitig adäquat behandelt worden, wäre es nicht zum Substanzmissbrauch gekommen und die Todesfälle wären vermieden worden. Die Personen wären auch nicht verstorben, hätten sie nur den Alkohol getrunken und auf Heroin verzichtet, oder umgekehrt nur Heroin genommen und auf Alkohol verzichtet. Die Todesfälle wären auch nicht eingetreten, wenn die Anwesenden rechtzeitig adäquat reagiert hätten.

$100 \%$ der UVT können in dieser Teilpopulation also mit Recht als vorzeitiger Tod infolge einer Depression, $100 \%$ als vorzeitiger Tod infolge von Alkoholkonsum, 100\% als vorzeitiger Tod infolge von Heroinkonsum und $100 \%$ als vorzeitiger Tod infolge von unterlassener Hilfeleistung der Anwesenden klassifiziert werden, was in Summe 400\% der UVT erklärt. Analog dazu gehen die 10 verlorenen Lebensjahre (UVL) auf das Konto der Depression, auf das Konto des Alkohols, auf das Konto des Heroins und auf das Konto der unterlassenen Hilfeleistung, was in Summe 40 UVL ergibt, obwohl pro Person tatsächlich nur 10 Jahre verloren gehen ${ }^{3}$.

Ganz offensichtlich führt die Summenbildung über unterschiedliche Ursachen hinweg infolge von Multikausalität sowohl bei UVT als auch bei UVL zu erheblichen Doppelzählungen. Je mehr Ursachen man berücksichtigt, desto mehr Doppelzählungen ergeben sich. In diesem Beispiel könnte man durch Intervention bei jedem einzelnen der vier Punkte zwar in allen Fällen UVT verhindern bzw. bewirken, dass pro Betroffenem die 10 UVL nicht

\footnotetext{
3 Da reale Populationen in der Regel nicht homogen sind, sondern sich aus unterschiedlichen Teilpopulationen mit unterschiedlichen Bedingungsgefügen zusammensetzen, ist das tatsächliche Ausmaß der Doppelzählungen in der Regel natürlich deutlich geringer als das maximale in diesem zu Demonstrationszwecken konstruierten Extrembeispiel.
} 
verloren gehen, man dürfte aber über die Ursachen keine Summen bilden.

Anschaulicher formuliert: Wenn x\% der Bevölkerung infolge ihres Rauchens und y\% infolge des Alkoholkonsums vorzeitig sterben, so bedeutet das nicht, dass man $\mathrm{x} \%$ plus $\mathrm{y} \%$ der Todesfälle durch Alkohol- plus Nikotinabstinenz retten könnte. Wenn Raucher x Lebensjahre und Alkoholkonsumenten y Lebensjahre durch ihr Konsumverhalten verlieren, so bedeutet das nicht, dass Personen, die trinken und rauchen, durch Verzicht auf beide Gewohnheiten x plus y Jahre gewinnen könnten.

Murray et al. [17] vertreten zwar, dass bei direkter Zuteilung, im Sinne des kategoriellen Ansatzes, Additivität gegeben ist, das trifft aber nur dann zu, wenn man, wie das in der internationalen Todesursachenstatistik erfolgt, Monokausalität erzwingt, indem man regelt, dass sich der Kodierer aus den existierenden Todesursachen nur eine aussuchen kann (vgl. dazu Abschnitt „Vermeidung von Multikausalität in der Todesursachenstatistik“).

Krankheiten sind eigentlich keine Todesursachen, sondern Mediatorvariablen

Als unabhängige Variablen in einem Experiment werden die vom Versuchsleiter direkt beeinflussten Größen bezeichnet. Diese haben einen Einfluss auf Mediatorvariablen, die letztlich die Ergebnisvariablen beeinflussen und über die der Zusammenhang zwischen den unabhängigen Variablen und den Ergebnisvariablen erklärt wird. Wenn nun z.B. ein Versuchsleiter durch geeignete Maßnahmen bewirkt, dass exzessive Alkoholkonsumenten in der Versuchsbedingung alkoholabstinent leben, während die Personen in der Kontrollbedingung unverändert weiter trinken, im Beobachtungszeitraum die Inzidenz alkoholbedingter Erkrankungen erfasst und die Ergebnisvariable „Lebenserwartung“ misst, so sind die Erkrankungen logisch gesehen Mediatorvariablen und keine unabhängigen Variablen, auf die man unmittelbar Einfluss nehmen könnte.

Im fiktiven Gedankenexperiment kann man Krankheiten zwar einfach wegdenken, im realistischen Gedankenexperiment, das einem realen Experiment nahe kommen soll, ist der Interventionspunkt aber immer dort anzusetzen, wo auch ein reales Experiment ansetzen würde, und das sind im gegenständlichen Fall Umweltbedingungen oder Verhaltensweisen, die Krankheiten begünstigen, und nicht die Krankheiten selbst. Der Umstand, dass Krankheiten in Zusammenhang mit Todesursachenstatistiken und Krankheitskostenrechnungen wie direkt beeinflussbare Größen und nicht als Auswirkungen interpretiert werden, führt, wie im nächsten Abschnitt gezeigt wird, zu Fehlschlüssen.

\section{Eindeutig alkoholverursachte Todesfälle müssen nicht vorzeitig sein und schädlicher Substanzkonsum kann lebensverlängernd wirken}

Wenn man in einem realen Experiment oder realistischen Gedankenexperiment den Alkoholkonsum in einer Versuchsbedingung verändert und die Auswirkungen mit dem unveränderten Alkoholkonsum in der Kontrollbedingung hinsichtlich Mortalität vergleicht, beobachtet man die Effekte aller unmittelbaren und mittelbaren Auswirkungen. Wenn man hingegen bestimmte alkoholverursachte Erkrankungen im Gedankenexperiment bloß wegdenkt, ignoriert man unter Umständen eine Reihe von relevanten Prozessen, die durch die Versuchsbedingung ausgelöst wurden.

Angenommen, ein depressiver Motorradfahrer, der seine Depression mit Alkohol behandelt [25], entwickelt infolge exzessiven Alkoholkonsums eine Leberzirrhose, ist immer müde und stirbt im Alter von 40 Jahren an dieser Zirrhose. Hier ist zwar ohne Frage sehr naheliegend, dass die Zirrhose das Leben des Betreffenden verkürzt hat, man kann aber im Einzelfall sicherlich nicht ausschließen, dass er z.B. ohne die zirrhosebedingte Müdigkeit öfter Motorrad gefahren wäre und damit bereits im Alter von 35 Jahren an einem Motorradunfall verstorben wäre oder mit 37 Jahren infolge seiner Depression Selbstmord verübt hätte. Dieser Person würde der Alkoholmissbrauch konkret fünf bzw. drei zusätzliche Lebensjahre bescheren, und man dürfte daher ihren Tod nicht als vorzeitigen Tod durch Alkoholmissbrauch bezeichnen, sondern müsste diese Person, so seltsam das auch klingt, als Gewinner des Alkoholmissbrauchs verkoden.

\section{Erfolgreiche Maßnahmen gegen ein Übel können das Übel vergrößern}

In einem realen Langzeitexperiment, in dem man Alkoholkonsum in der Versuchsbedingung auf einen moderaten Konsum oder auf Abstinenz reduzieren würde, würde die Analyse zwangsläufig auch unerwünschte Mediatoreffekte auf die Mortalität abbilden. Beispielsweise ist bei einer Verknappung oder Verteuerung von Alkohol damit zu rechnen, dass die illegale Produktion von weit giftigeren Produkten steigt, dass organisierte Kriminalität wie zu Zeiten der US-amerikanischen Prohibition in den 20er-Jahren des letzten Jahrhunderts zunimmt oder verstärkt funktionale Äquivalente wie illegale Drogen oder Medikamente zum Einsatz kommen. Das Ergebnis in Hinblick auf die Mortalitätsrate wäre die Zahl jener, die infolge der Maßnahmen nicht vorzeitig an alkoholbedingten Erkrankungen gestorben sind, abzüglich jener, die an den Folgen toxischer Alkoholprodukte zusätzlich gestorben sind, abzüglich jener, die infolge der gestiegenen Kriminalität vorzeitig verstorben sind, sowie abzüglich jener, die infolge des Konsums funktionaler Äquivalente vorzeitig verstorben sind.

Mit großer Wahrscheinlichkeit wäre die Gesamtbilanz hinsichtlich Mortalität erheblich weniger positiv als jene, die sich aus $\mathrm{Ge}$ dankenexperimenten ergeben, die den Alkoholkonsum einfach wegdenken, ohne mögliche Nebenwirkungen der Maßnahmen zu berücksichtigen.

\section{Vermeidung von Multikausalität in der Todesursachenstatistik}

Wie oben ausgeführt wurde, ist es aus einer praktischen Perspektive nicht unproblematisch, Krankheiten als Todesursachen anzugeben, weil es sich bei Krankheiten nicht um direkt beeinflussbare Größen, sondern um Auswirkungen von Umweltbedingungen und Verhaltensweisen handelt, die Krankheiten begünstigen. Aber selbst, wenn man mangels Alternativen akzeptiert, dass Krankheiten als Todesursachen gesehen werden, steht man vor dem Problem, dass die meisten Menschen in unmittelbarer Todesnähe an vielen Krankheiten leiden, was in hohem Maße Multikausalität bedingt. Dieses Problem wurde international behoben, indem 
man definitorisch festlegte, dass für jeden Todesfall nur eine einzige Todesursache angegeben werden darf, also monokausal zu konzipieren ist. Konkret steht jeder Kodierer nun vor dem Problem, aus einer Kausalkette von Todesursachen jenes Grundleiden auszuwählen, das am ehesten zum Tod geführt hat, wobei er erschwerend dabei auch noch auf jene Informationen angewiesen ist, die im Totenschein angegeben wurden [26]. Diese Aufgabenstellung ist verständlicherweise in den meisten Fällen nicht adäquat und eindeutig zu lösen. Soll man z. B. bei einem Depressiven, der seine Depression mit Alkohol bekämpfte und in der Folge alkoholabhängig wurde, der infolge einer Leberzirrhose eine Ösophagusvarizenblutung im Krankenhaus gerade noch überlebt, dort aber an einer Lungenentzündung stirbt, „Tod infolge Depression“, „Tod infolge von Alkoholismus“ oder „Tod infolge einer Lungenentzündung" klassifizieren? Dazu kommt noch das Problem, dass die in Zusammenhang mit der Abschätzung der Alkoholismusprävalenz bedeutende Diagnose Leberzirrhose in diesem Fall in der Statistik gar keinen Niederschlag finden würde.

Die Festlegung auf eine einzige Todesursache ist in den meisten Fällen nur über recht willkürliche Entscheidungen erzielbar und führt zwangsläufig zur Ausbildung von unterschiedlichen impliziten Konventionen bei unterschiedlichen Kodierern. Infolge der sich daraus ergebenden Reliabilitäts- und Validitätsprobleme sind Vergleiche über die Zeitachse innerhalb von Ländern sowie Vergleiche zwischen unterschiedlichen Ländern nur sehr bedingt sinnvoll.

Das quantitative Ausmaß der Problematik unterstreicht ein Blick auf einige ausgewählte internationale Ergebnisse [27]. Bezogen auf das Jahr 1995 gab es in Finnland unter Männern zehnmal so viele als explizit alkoholbedingt ausgewiesene Todesfälle als in Irland, obwohl in Irland um 50\% mehr Alkohol konsumiert wurde. In Finnland wurden im selben Jahr zehnmal so viele Leberzirrhosen als alkoholbedingt ausgewiesen wie in Österreich, obwohl die Österreicher um ein Drittel mehr Alkohol tranken als die Finnen. Die Gesamtleberzirrhoserate war in Österreich in ebendiesem Jahr bei Männern fünfmal so hoch wie in Griechenland, obwohl die Österreicher nur unwesentlich mehr Alkohol tranken als die Griechen.

\section{Methodische Probleme der Messbarkeit des Konsums}

Probleme mit der Verlässlichkeit der Daten gibt es aber nicht nur bei der Todesursachenstatistik. Untersuchungen zum Zusammenhang zwischen Substanzkonsum, Erkrankungsrisiko und Tod basieren neben Schätzungen des Gesamtkonsums in der Regel auf subjektiven Angaben zum Konsumverhalten. Und auch dort, wo relativ harte Daten vorzuliegen scheinen - beispielsweise bei Gesamtschätzungen des Alkoholkonsums basierend auf Angaben zur Produktion und zum Verkauf -, werden andere Quellen wie illegale Produktion und grenzüberschreitender Schmuggel in der Regel nicht berücksichtigt. Schätzungen aus Selbstangaben unterliegen zum einen Fehlern, die sich aus der Auswahl der Personen und dem Studiendesign ergeben, und zum anderen Fehlern, die mit den Angaben der Befragten zum Konsum selbst zu tun haben. So basieren Befragungen zum Alkoholkonsum auf Stichprobendesigns, bei denen Personengruppen mit hohem Konsum systematisch unterrepräsentiert sind. Zum anderen weisen methodische
Studien auf eine systematische Unterschätzung von Häufigkeitsund Mengenangaben des Alkoholkonsums hin [28, 29]. Ursachen dafür ergeben sich aus der Schwierigkeit, ein subjektiv sehr variables Verhalten auf Durchschnittsangaben zu reduzieren und aus einem normorientierten Verhalten der befragten Personen. Starke Trinker neigen dazu, ihre Angaben an sozial erwünschten Normen des mäßigen Trinkens zu relativieren und daher ihren Konsum zu unterschätzen. Vergleiche mit Verbrauchszahlen zeigen, dass Schätzungen des Alkoholkonsums aus Surveydaten nur $60 \%$ oder weniger der offiziellen Produktions- und Verkaufsstatistiken ausmachen $[30,31]$.

Ein weiteres Problem ergibt sich daraus, dass sich Analysen des Zusammenhangs zwischen Konsumverhalten und den relevanten Ergebnisvariablen in den meisten Fällen auf Konsumwerte stützen, die oft viele Jahre vor dem Eintritt der interessierenden Ereignisse erhoben wurden. Damit die einmalig erfassten Konsumwerte valide Prädiktoren des Zusammenhangs zwischen Konsum und - in unserem Fall - des Todes sind, bedarf es der eher unrealistischen Annahme, dass das Konsumverhalten über die Zeit stabil geblieben ist [32, 33].

Maßnahmen, die eine bestimmte Erkrankung verhindern, begüinstigen zwangsläufig andere Erlkrankungen

In der Regel wird jede Zunahme einer bestimmten Todesursache in den offiziellen Statistiken als Alarmzeichen interpretiert. Sieht man einmal vom Problem ab, dass es angesichts der Multikausalität kaum sinnvoll ist, nur eine bestimmte Ursache pro Todesfall als Hauptursache auszuweisen, tritt noch ein weiteres gravierendes logisches Problem auf. Man kann den Tod nur aufschieben, aber nie wirklich verhindern. Der Wegfall einer bestimmten Todesursache und der mit dieser Ursache kausal verbundenen Krankheiten bewirkt zwangsläufig die Zunahme anderer Krankheiten. Eine hohe Zahl an Krebstoten und eine hohe Rate an Menschen, die an Herz-Kreislauf-Erkrankungen versterben, ist zumindest in industrialisierten Ländern, in denen die Lebenserwartung steigt, kein negatives, sondern ein positives Zeichen. Ein funktionierendes Sozialsystem, gute Gesundheitsversorgung und optimale Maßnahmen zur Unfallverhütung lassen die Menschen so alt werden, dass die klassischen Alterskrankheiten an Stellenwert gewinnen.

Wie Rothman und Greenland [15] betonen, ist es beim Vergleich von Mortalitätsraten grundsätzlich nicht möglich zu entscheiden, ob die Exposition zu einem bestimmten Faktor die Prognose für eine bestimmten Todesursache erhöht oder die Prognose für eine konkurrierende andere Todesursache verringert. Auch eine hohe Leberzirrhoserate bedeutet nicht unbedingt, dass es in einem Land mehr Alkoholprobleme gibt als in einem anderen Land mit einer niedrigeren Rate. Alkoholiker, die gar nicht alt genug werden, um eine Leberzirrhose entwickeln zu können, sowie Alkoholiker, die, bevor ihre Leberzirrhose als Todesursache manifest werden kann, an vermeidbaren Infektionskrankheiten oder Unfällen sterben, tauchen in der Todesursachenstatistik nicht im Zusammenhang mit einer Leberzirrhose auf. Bei einer naiven Interpretation der Todesursachenstatistik kann man leicht zu dem irrigen Schluss kommen, dass jede noch so gefährliche Noxe gleichzeitig protektiv gegen andere Krankheiten wirkt. 
Vergleiche mit Referenzpopulationen

\section{UVT Rohwerte}

Die US-amerikanischen Centers for Disease Control and Prevention geben an, dass im Zeitraum von 1995 bis 1999 in den USA jährlich 442398 Personen vorzeitig am Tabakkonsum [11] und im Jahre 200175766 Personen vorzeitig am Alkoholkonsum [34] starben, ohne als Referenzzahl die pro Jahr insgesamt verstorbenen Personen oder die Wohnbevölkerung anzugeben. Derartige Mortalitätskoeffizienten sind aber nur in Relation zu Bezugsgrößen inhaltlich interpretierbar. Mögliche und übliche Bezugsgrößen für die UVT sind die Anzahl der Verstorbenen, die Wohnbevölkerung und die Standardwohnbevölkerung.

UVT bezogen auf die im Beobachtungszeitraum Verstorbenen Bezieht man die UVT auf die Zahl aller im Beobachtungszeitraum Verstorbenen, dann hat man den Einfluss Lebenserwartung der Bevölkerung rechnerisch eliminiert und vergleicht damit nur die relativen Anteile der Todesursachen. Bei dieser Betrachtungsform kann unter Berücksichtigung einer monokausalen Todesursachenstatistik jede Todesursache nur dann abnehmen, wenn gleichzeitig andere Todesursachen zunehmen. Man ist mit dem logischen Phänomen konfrontiert, dass die Zunahme einer bestimmten Ursache zwangsläufig zur Abnahme anderer Ursachen führen muss, was isoliert kaum interpretierbar ist und Fehlschlüsse geradezu nahe legt.

\section{UVT bezogen auf die Wohnbevölkerung}

Bezieht man die Summe aller UVT pro Ursache nicht wie im oben genannten Fall auf die Zahl der Verstorbenen, sondern auf die Wohnbevölkerung, so bleibt das im letzten Abschnitt genannte logische Grundproblem weiterhin bestehen, wird aber dadurch verkompliziert, dass nun auch noch die durchschnittliche Lebenserwartung in die Maßzahlen einfließt.

\section{UVT bezogen auf die Standardwohnbevölkerung}

Indem man die Ergebnisse auf eine Standardbevölkerung bezieht, was man eigentlich immer tun sollte, macht man Vergleiche zwischen unterschiedlichen Epochen und zwischen unterschiedlichen Ländern erst möglich und sinnvoll. Es muss einem aber bewusst sein, dass man auch damit das genannte logische Problem nicht in den Griff bekommt und dass man über die Wahl der Standardbevölkerung maßgeblichen Einfluss auf die Ergebnisse nimmt. Überwiegen in der Bevölkerung ältere Menschen, so erhalten Alterserkrankungen viel Gewicht, und überwiegen jüngere Menschen, so legt man implizit den Fokus auf Erkrankungen, die bei Jugendlichen gehäuft auftreten.

\section{UVL Gesamtzahlen}

In der Praxis sind UVL Rohwertsummen allein wegen der hohen Zahlen sehr beliebt. Die CDC berichtet beispielsweise, dass im Zeitraum von 1995 bis 1999 in den USA jährlich 5616385 Lebensjahre als Folge des Tabakkonsums [11] und im Jahre 2001 2279322 Lebensjahre als Folge des Alkoholkonsums [34] verloren gingen, ohne als Referenzzahl die pro Jahr insgesamt verstorbenen Personen oder die Wohnbevölkerung anzugeben. Wie schon in Zusammenhang mit den UVT betont, sind derartige Angaben ohne Bezug zu Referenzzahlen nicht sinnvoll interpretierbar.

\section{UVL pro Indexfall}

Eine Möglichkeit, Ergebnisse darzustellen, besteht darin, die UVL auf Indexfälle zu beziehen, z. B. darauf, wie viele Lebensjahre Raucher durch den Nikotinkonsum verlieren. Infolge der Multikausalität ist es aber, wie bereits ausgeführt, nicht sinnvoll, die Summen der verlorenen Lebensjahre über verschiedene Todesursachen wie Rauchen und Alkoholkonsum aufzusummieren. Eine Ausnahme von dieser Regel stellt das Aufsummieren der kausal attribuierbaren Anteile basierend auf den Todesursachen der offiziellen Todesursachenstatistik dar. Diese Summe ist zumindest formal sinnvoll, wenngleich man die immanenten Probleme der per Definition monokausal eingerichteten Todesursachenstatistik und die empirischen Probleme bei der Bestimmung der KAA hier nicht übersehen sollte.

\section{UVL pro Gestorbenen}

Kennt man die Zahl der Indexfälle, kann man aus der Zahl der UVL pro Indexfall recht einfach auf die UVL pro Person umrechnen. Zum Beispiel, wie viele Lebensjahre der Durchschnittsperson durch Nikotinkonsum verloren gehen.

\section{DALYs und QUALYs}

In Zusammenhang mit den UVL sind Lebensqualitätsüberlegungen im Sinne von qualitätsadjustierten bzw. behinderungsadjustierten Lebensjahren von Bedeutung (vgl. den Beitrag von Gmel und Rehm in diesem Heft). Auf diese Aspekte wird in diesem Aufsatz nicht eingegangen.

\section{Unterteilung nach Unmittelbarkeit der Todesfolge}

Wie oben ausgeführt, lassen sich akute Todesfälle, die unmittelbar im Zusammenhang mit Substanzkonsum auftreten, sinnvoll als UVT interpretieren, während für Todesfälle als langfristige Folgen des Substanzkonsums UVL geeigneter sind. In diesem Sinne sollten Überdosierungen plus andere substanzverursachte Verkehrs-, Arbeits- und Freizeitunfälle über UVT und Todesfälle in Folge langfristiger Krankheiten über UVL erfasst werden. Wenn ein Depressiver sein Problem über Substanzmissbrauch in den Griff bekommen möchte, letztlich Selbstmord begeht, ist sein Tod nicht als substanzbedingt zu werten. Wenn umgekehrt jemand infolge des Substanzmissbrauchs eine Depression entwickelt, ist es unerheblich, ob er sich mit einem Revolver oder mit Heroin umbringt.

Die von der Europäischen Beobachtungsstelle für Drogen und Drogensucht [35] vorgeschlagene Unterteilung in direkte Todesfälle, bei denen eine Überdosierung kurzfristig zum Tode führte, und in indirekte Todesfälle, bei denen der Tod zwar in Zusammenhang mit dem Substanzkonsum steht, aber nicht über eine Überdosierung erfolgt (vgl. dazu den Beitrag von Busch in diesem Heft), erscheint im Lichte dieser Überlegungen als suboptimal. Selbstmorde mit Substanzen werden dabei als direkte Substanztote gezählt und substanzbedingte Unfälle als indirekte. Auch die von Uhl [27] vorgeschlagene Unterteilung von Substanztod in unabsichtliche Überdosierungen und substanzbedingte Unfälle, in Todesfälle, verursacht durch substanzbedingte Erkrankungen, und in Todesfälle von Süchtigen, Missbrauchern und Konsumenten ist aus dieser Warte zu präzisieren. Nur im ersteren Fall (Überdosierungen und substanzbedingte Unfälle) ist die Erfassung über UVT tatsächlich sinnvoll. 
Es macht einen großen Unterschied, ob die Auswirkungen einer Substanz von der Summe der konsumierten Einheiten abhängt, von der aktuellen durchschnittlichen Belastung, von der aktuellen Maximalbelastung oder von der Zeit, wie lange die Belastungen zurückliegt. Es gibt Modelle, nach denen das Risiko, an einer Leberzirrhose zu erkranken, von der Gesamtmenge des jemals konsumierten Alkohols abhängt [36]. Andere Modelle gehen davon aus, dass die Krankheitsgefährdung nach Abstinenzbeginn rasch abnimmt und irgendwann das Niveau von Nichtkonsumenten erreicht. Wieder andere Modelle setzen ausschließlich die kardioprotektive Wirkung des Alkohols akut an. Das Argument, dass sich Alkoholkonsum erst ab einem bestimmten Alter protektiv gegen koronare Herzerkrankungen auswirken kann, weil es davor keine relevante Gefährdung gibt, an Herzerkrankungen zu sterben, impliziert eine Akutwirkung. Sollte es hingegen der Fall sein, dass Alkohol die Verkalkung von Gefäßen langfristig verringert, wäre der Effekt kumulativ.

\section{Schlussfolgerungen}

Die Berechnung von ursachenbezogenen Mortalitätskoeffizienten (UMK) erfordert eine klare Definitionen der Modellstruktur, d.h. der zugrunde liegenden Definitionen, Konzepte und deren logischer Bezüge, klare Vorstellungen der Messbarkeit, d.h. wie die für die Berechnung notwendigen empirischen Teilgrößen theoretisch erhoben werden können, wie reliabel und valide die derzeit verwendeten Maßzahlen sind, und welche Schlussfolgerungen man basierend auf die so errechneten UMK logisch korrekt anstellen kann. Im Zusammenhang mit dem ersten Aspekt ergibt sich eine Fülle von gravierenden Problemen. Diese beinhalten insbesondere die Unmöglichkeit, ohne kontrollierte Experimente Kausalität eindeutig zu belegen, die damit zusammenhängende Notwendigkeit, den Einfluss von relevanten Drittvariablen auf die Zusammenhänge zu entdecken und zu kontrollieren, sowie die Problematik der Multikausalität. Hier sollte die Suche nach logischen Inkonsistenzen und dem Gegenstand angemessenen Modellen aktiv betrieben werden.

Im Zusammenhang mit der empirischen Erfassung der Teilgrößen ergibt sich das Problem, dass ein aus der Warte der Berechnung von UMK optimales Erfassungssystem so kostenaufwändig wäre, dass man dieses angesichts des Nutzens der UMK kaum rechtfertigen könnte. Deswegen wird in hohem Maße auf Datenbestände zurückgegriffen, die zwar routinemäßig, aber in der Regel mit ganz anderen Zielsetzungen erhoben werden. Hier ist es nötig, die sich durch schlechte Datenqualität ergebenden Ungenauigkeiten offen zu diskutieren, auch wenn es für Experten nicht en vogue ist, die bekannten Probleme ihrer Produkte explizit nach außen zu tragen.

Im Zusammenhang mit den zulässigen Schlussfolgerungen ist zu erwähnen, dass die korrekte Interpretation die Kenntnis der Details des theoretischen Modells und Informationen über die Datenqualität und deren Implikation voraussetzt. Über diese Voraussetzungen verfügen zwar jene Experten, die die Berechnungen anstellen, aber kaum je die Nutznießer der Daten, seien es die an der Berechnung unbeteiligten Experten, Politiker, Vertreter der Medien oder die Öffentlichkeit. So ist jenen Autoren, die UMK berechnen, zwar klar, dass die Zahl der durch Alkoholabstinenz vermeidbaren Todesfälle eine fiktive Größe darstellt, die praktisch nicht erreichbar ist, weil es kaum möglich ist, Alkoholkonsum in der Gesellschaft völlig zu verhindern. Sie sind sich dessen bewusst, dass die dafür erforderlichen Experimente in einer aufgeklärten Gesellschaft ethisch nicht zu rechtfertigen sind, und sie wissen, dass der Wegfall des Alkohols den Konsum von Ersatzstoffen oder Ersatzhandlungen begünstigen würde, deren Auswirkungen auf die Mortalität mit hoher Wahrscheinlichkeit negativ sein würden. Den Autoren ist klar, dass der Begriff „vorzeitig“ im Zusammenhang mit Tod alles - von wenigen Augenblicken bis mehrere Jahrzehnte - bedeuten kann, dass alle Berechnungen auf unsichere Daten aufbauen und eine Reihe von unbeweisbaren bzw. unbewiesenen Annahmen voraussetzen.

Die Anwender können aber aus den Ergebnissen nur dann adäquate Schlussfolgerungen ziehen, wenn ihnen diese Probleme explizit zur Kenntnis gebracht werden. Aus diesem Grund erscheint es zweckmäßig, den Anwendern neben der Warnung, die Verlässlichkeit der Resultate nicht zu überschätzen, auch Vorschläge zu machen, welchem UMK in welchem Zusammenhang der Vorzug zu geben ist. In diesem Sinne kann man sagen, dass die Zählung von UVT eigentlich nur sinnvoll ist, wenn es sich dabei um zeitlich unmittelbare Auswirkungen eines Risikofaktors handelt, wie z.B. bei Unfällen im Sinne von Überdosierungen und Verkehrsunfällen, und auch hier nur, wenn der auf den Substanzkonsum kausal attribuierbare Anteil korrekt geschätzt wurde. Selbstmorde mit oder ohne Substanzüberdosierungen sind bei Substanzmissbrauchern keine Akutauswirkungen, sondern Folge einer Depression, die zum Substanzmissbrauch geführt hat, oder Folge einer Depression, die als Folge des Substanzmissbrauchs entstanden ist.

Bei Selbstmorden und anderen nicht unmittelbaren Folgen eines Risikofaktors ist es sinnvoller, die Effekte über UVL zu erfassen. Auch hier muss man darauf abzielen, nur jene verlorenen Lebensjahre zu werten, die ohne Substanzmissbrauch nicht entstanden wären. Bei Depressiven, die Substanzmissbrauch im Sinne von Selbstmedikation einsetzten, kann man nicht einmal ausschließen, dass der Substanzmissbrauch tatsächlich lebensverlängernd gewirkt hat.

Generell gilt sowohl für UVT als auch für UVL, dass diese Größen selbst im Fall, dass es gelänge, über eine vollständige Ausschaltung aller relevanten Drittvariablen den kausal attribuierbaren Anteil optimal wie bei einem realen, gut geplanten Experiment zu schätzen, die Effekte unterschiedlicher Risikofaktoren nicht addierbar sind. Schließlich sollte noch einmal betont werden, dass die empirischen und logischen Grundlagen für die Berechnung der UVT und UVL keinesfalls so gut sind, wie man angesichts der Sicherheit, mit der derartige Zahlen in der Regel präsentiert werden, annehmen würde. Während man als Nutzer bei der Interpretation von UMK sehr vorsichtig sein sollte, ist es Aufgabe der Experten, die empirische und logische Basis bei der Berechnung dieser Koeffizienten systematisch zu verbessern. 


\section{Literatur}

${ }^{1}$ Bühringer G, Augustin R, Bergmann E et al. Alcohol consumption and alcohol-related problems in Germany. Göttingen: Hogrefe \& Huber, 2002

${ }^{2}$ John U, Hanke M. Tabakrauch-attributable Mortalität in den deutschen Bundesländern. Gesundheitswesen 2001; 63 (6): 363 - 369

3 John U, Hanke M. Tobacco- and alcohol-attributable mortality and years of potential life lost in Germany. European Journal of Public Health 2003; 13 (3): $275-277$

${ }^{4}$ Rehm J, Taylor B, Patra J. Volume of alcohol consumption, patterns of drinking and burden of disease in the European Region. Addiction 2006; 101 (8): 1086 - 1095

${ }^{5}$ Ridolfo B, Stevenson C. The quantification of drug-caused mortality and morbidity in Australia 1998. Canberra, Australia: Australian Institute of Health and Welfare, 2001

${ }^{6}$ Single E, Robson L, Rehm J et al. Morbidity and mortality attributable to alcohol, tobacco, and illicit drug use in Canada. American Journal of Public Health 1999; 89 (3): 385-390

${ }^{7}$ English DR, Holman SDJ, Milne E et al. The quantification of drug caused morbidity and mortality in Australia 1995. Canberra, Australia: Commonwealth Department of Human Services, 1995

${ }^{8}$ Hartnoll R. Drugs and drug dependence: linking research, policy and practice background Paper for the Pompidou Group's strategic conference on connecting research, policy and practice, 6-7 April. Strasbourg: Council of Europe Publishing, 2004

${ }^{9}$ Murray CJL, Salomon JA, Mathers CD et al. Summary measures of population health: concepts, ethics, measurement and applications. Geneva: WHO Nonserial Publication, 2002

${ }^{10}$ Schultz JM, Rice DP, Parker DL. Alcohol-related mortality \& years of potential life lost-U.S. - United States 1987. Morbidity and Mortality Weekly Report 1990; 39 (11): 173-178

${ }^{11}$ Centers for Disease Control and Prevention (CDC). Annual smoking attributable mortality, years of potential life lost, and economic costs - United States, 1995 - 1999. Morbidity and Mortality Weekly Report 2002; 51 (14): 300-303

${ }^{12}$ Gmel G, Rehm J. Harmful alcohol use. Alcohol Research \& Health 2003; 27,1: $52-62$

${ }^{13}$ Centers for Disease Control and Prevention (CDC). About ARDI (Alcohol-related disease impact).; http://apps.nccd.cdc.gov/ardi/HomePageaspx (24.9.2006)

${ }^{14}$ Ezzati M, Lopez AD, Rodgers A et al. Selected major risk factors and global and regional burden of disease. The Lancet 2002; 360: $1347-1360$

${ }^{15}$ Rothman KJ, Greenland S. Modern epidemiology. 2nd edition. Philadelphia: Lippincott, Williams \& Wilkins, 1998

${ }^{16}$ Murray CJL, Salomon JA, Mathers C. A Critical Examination of Summary Measures of Population Health. Bulletin of the World Health Organization 2000; 78: 981 - 994

17 Murray C, Ezzati M, Lopez A et al. Comparative quantification of health risks: conceptual framework and methodological issues (Chapter 1). In: Ezzati M, Lopez A, Rodgers A, Murray C (Hrsg). Comparative quantification of health risks: global and regional burden of disease due to selected major risk factors. Geneva: WHO, 2004

${ }^{18} \mathrm{Uhl}$ A. Darstellung und kritische Anallyse von Kostenberechnungen im Bereich des Substanzmissbrauchs. Sucht 2006; 52 (2): 121-132
${ }^{19}$ Mooney G, Irvig L, Leeder S. Priority setting in health care: unburdening from the burden of disease. Australian and New Zealand Journal of Public Health 1997; 21 (7): 680-681

${ }^{20} \mathrm{Uhl}$ A. How to camouflage ethical questions in addiction research. In: Fountain J, Korf D (Hrsg). The social meaning of drugs - Research from Europe. Oxford: Radcliffe, in Druck

${ }^{21}$ Kuratorium für Verkehrssicherheit. Unfallstatistik 2004. Reihe Verkehr in Österreich: Heft 37. Wien: Kuratorium für Verkehrssicherheit, 2005

${ }^{22}$ Borkenstein RF, Crowther RF, Shumate RP et al. The role of the drinking driver in traffic accidents. Department of Police Administration, Indiana University, 1964. Reprinted. Blutalkohol 1974; 11: 1 - 131

${ }^{23}$ MacMahon B. The quantification of alcohol-caused morbidity and mortality in Australia: a critique. Medical Journal of Australia 1992; 157: $557-560$

${ }^{24}$ Doll R, Peto R. The causes of cancer. New York: Oxford University Press, 1981 (zit. in Rothman, \& Greenland 1998)

${ }^{25}$ Bell CM, Khantzian EJ. Drug use and addiction as self-medication: a psychodynamic perspective. In: Gold M, Slaby A (Hrsg). Dual diagnosis in substance abuse. New York: Dekker Press, 1991

${ }^{26}$ Leitner B. Methodik der Österreichischen Todesursachenstatistik. Allgemeine Informationen und Richtlinien zum Ausfüllen des Formblattes. Wien: Statistik Austria, 2004

27 Uhl A. Was sind eigentlich 'Drogenopfer'? In: Beubler E, Haltmayer $\mathrm{H}$ Springer A (Hrsg). Opiatabhängigkeit Interdisziplinäre Aspekte für die Praxis. Wien: Springer, 2003

${ }^{28}$ Lemmens P, Knibbe RA, Tan F. Weekly recall and diary estimates of alcohol consumption in a general population survey. Journal of Studies on Alcohol 1988; 49: $131-135$

${ }^{29}$ Midanik LT. Comparing usual quantity/frequency and graduated frequency scales to assess yearly alcohol consumption: results from the 1990 US National Alcohol Survey. Addiction 1994; 89: 407-412

${ }^{30} \mathrm{Rehm}$ J. Measuring quantity, frequency, and volume of drinking. Alcoholism: Clinical and Experimental Research 1998; 22 (Suppl): S4 -S14

${ }^{31}$ Kraus L, Bauernfeind R, Bühringer G. Epidemiologie des Drogenkonsums: Ergebnisse aus Bevölkerungssurveys 1990 bis 1996 (Schriftenreihe des Bundesministeriums für Gesundheit 107). Baden-Baden: Nomos Verlagsgesellschaft, 1998

${ }^{32}$ Sempos CT, Flegal KM, Johnson CL et al. Issues in the long-term evaluation of diet in longitudinal studies. Journal of Nutrition 1993; 123: $405-412$

${ }^{33}$ Rehm J, Gmel G, Smpos CT et al. Alcohol-related morbidity and mortality. Alcohol Research \& Health 2003; 27 (1): 39-51

${ }^{34}$ Centers for Disease Control and Prevention (CDC). Alcohol-attributable deaths and years of potential life lost - United States, 2001. Morbidity and mortality weekly report 2004; 53 (37): 866-870

35 European Monitoring Centre for Drugs and Drug Addiction. The DRDStandard 3.0. EMCDDA standard protocol for the EU member states to collect data and report figures on the Key Indicator Drug-Related Deaths by the standard Reitox table. Lisbon: European Monitoring Centre for Drugs and Drug Addiction, 2002

${ }^{36}$ Lelbach WK. Dosis-Wirkungs-Beziehung bei Alkohol-Leberschäden. Deutsche medizinische Wochenschrift 1972; 97: 1435-1436 